\title{
High Capacity Converged Passive Optical Network and RoF-based 5G+ Fronthaul Using 4-PAM and NOMA-CAP Signals
}

\author{
Samael Sarmiento, José Manuel Delgado Mendinueta, Member, IEEE, José Antonio Altabás, \\ Salvatore Spadaro, Senior Member, IEEE, Satoshi Shinada, Member, IEEE, Hideaki Furukawa, \\ Member, IEEE, Juan José Vegas Olmos, Senior Member, IEEE, José Antonio Lázaro, Member, IEEE, \\ and Naoya Wada, Member, IEEE
}

\begin{abstract}
Network architectural changes to satisfy all the $\mathbf{5 G +}$ mobile network specifications and requirements are necessary due to the popularization of streaming and cloud applications on omnipresent portable devices. The combination of massive installation of micro-cell antenna sites with the cloud access radio network (C-RAN) architecture has recently been nominated as a promising technology for high-capacity mobile fronthaul links, albeit at a high cost. An alternative approach for next-generation fronthaul networks is to utilize the already deployed passive optical networks (PONs) where wireless and wired services may coexist in a converged manner. Non-orthogonal multiple access (NOMA) modulation with multi-band carrierless amplitude and phase modulation (NOMA-CAP) has recently been investigated as a promising $5 \mathrm{G}+$ modulation format candidate to increase the capacity and flexibility of future mobile networks. Here, we experimentally demonstrate the convergence of a NOMA-CAP wireless waveform with a single-carrier wired signal in a PON scenario using radio-over-fiber (RoF) technology. Specifically, fifteen NOMA-CAP bands, with two NOMA power levels to double the capacity, transmit $15 \mathrm{~Gb} / \mathrm{s}$ multiplexed with a digital 10 Gb/s four-level pulse amplitude modulation (PAM-4) signal for downlink application. Two converged system implementations have been considered, first using electrical frequency division multiplexing (EFDM) and secondly using the hybrid EFDMwavelength division multiplexing (EFDM-WDM). Successful transmission through a $25 \mathrm{~km}$ span of standard single-mode fiber is achieved with negligible transmission penalty for both proposed converged solutions.
\end{abstract}

Index Terms-Mobile fronthaul convergence, multi-band carrierless amplitude and phase modulation, non-orthogonal multiple access, optical access networks, radio-over-fiber, successive interference cancellation.
Manuscript received MM DD, AA; revised MM DD, AA; accepted MM DD, AA. This work was supported in part by ALLIANCE (TEC2017-90034C2-2-R) project co-funded by FEDER, the European Union's Horizon 2020 research and innovation programme under grant agreements no 761989 and 871900 (5G-PHOS and 5G-COMPLETE), as well as MINECO FPI-BES-2015074302. (Corresponding author: Samael Sarmiento.)

Samael Sarmiento, Salvatore Spadaro and José Antonio Lázaro are with the School of Telecommunications Engineering, Polytechnic University of Catalonia, Barcelona 08034, Spain (e-mail:, samael.sarmiento@tsc.upc.edu; spadaro@tsc.upc.edu; jose.lazaro@tsc.upc.edu).

\section{INTRODUCTION}

$\mathrm{O}$ wing to the immense increment of the network traffic due to novel multimedia streaming services and cloud services on personal devices, combined with future extensive machineto-machine communication [1], network operators envision to deliver both broadband wireless and wireline services to final users through the already-deployed access network infrastructure. This approach minimizes both the capital expenditure (CAPEX) and the operation expenditure (OPEX) $[2,3]$. Moreover, already deployed passive optical networks (PONs) are the most competitive solution to serve as mobile fronthaul, especially in cloud access radio network (C-RAN) scenarios where flexibility, low latency and high capacity are compulsory [4]. Furthermore, deployed PONs can be upgraded in a cost-effective way to concurrently support both wired and wireless services in a converged manner.

C-RAN considers the division of the traditional base station into several only-transmitting, low-complexity and cheap remote radio heads (RRHs) and a single cloud-hub base band unit (BBU) located at the central office (CO), where complex processing is centralized. Open base station standard initiative (OBSAI) and common public radio interface (CPRI) are the transmission techniques used in $4 \mathrm{G}$ fronthaul networks. Nevertheless, these interfacing techniques are inadequate for high capacity and massive $5 \mathrm{G} / 5 \mathrm{G}+$ mobile communication services where high spectral efficiency is imperative. Ethernetbased CPRI (e-CPRI), which performs a digitization of the RF signal, is used in current $5 \mathrm{G}$ fronthaul network rollouts due to its higher efficiency, flexibility, and its low quantization resolution requirement. Moreover, e-CPRI is compatible with both telecom and enterprise networks enabling Xhaul

José Manuel Delgado Mendinueta, Satoshi Shinada, Hideaki Furukawa and Naoya Wada are with the Photonic Network System Laboratory, National Institute of Information and Communication Technology (NICT), 4-2-1 NukuiKitamachi, Koganei, Tokyo 184-8795, Japan.(e-mail: mendi@nict.go.jp, sshinada@nict.go.jp, furukawa@nict.go.jp, wada@ nict.go.jp).

José Antonio Altabás is with Bifrost Communications, Scion DTU, Akademivej Bygnig 381, 2800 Kgs Lyngby, Denmark, and the Aragon Institute of Engineering Research, University of Zaragoza, Zaragoza 50018, Spain (email: jaltabas@unizar.es).

Juan José Vegas Olmos is with Mellanox Technologies, Yokneam, 20692, Israel (e-mail: juanj@mellanox.com). 
deployments [5]-[7]. Despite all these benefits, e-CPRI requires significant digital signal processing (DSP) resources in the RRH that increases the system power consumption. To solve this problem, radio-over-fiber ( $\mathrm{RoF}$ ) technique has recently been proposed since it conserves bandwidth and simplifies the interface design of the RRHs, digitization and format conversion are not required [8]-[11]. Moreover, working in Cband with dense WDM (DWDM) technology can enable network densification which is not achievable in 5G networks [12].

In the next generation RANs, the design of the mediumaccess layer is fundamental to enhance the network capacity, the densification and to allocate the available resources in a dynamic way. Recently, non-orthogonal multiple access (NOMA), which essentially consists of multiplexing two or more users in the power domain, has been investigated as an alternative solution for addressing the aforementioned requirements of network capacity, densification and flexibility in future 5G+ mobile networks [13]. Moreover, NOMA can serve multiple users with different channel conditions simultaneously, providing user fairness enhancement. Successive interference cancellation (SIC) must be conducted at the receiver for the multi-user signal separation [14], which increases the receiver complexity for a high number of users. To mitigate this issue, redundancy can be added to the users' signals through spectrum spreading or coding techniques since it facilitates the user's signal separation, but decreasing the spectral efficiency [15]. Alternatively, SIC can be implemented with moderate complexity, without the need for spreading/coding redundancy, by limiting the number of power-multiplexed users to two and the constellation alphabet to four symbols, as demonstrated in [16]. Moreover, SIC with moderate complexity can be implemented using available technology with a tolerable increase of the energy consumption [17].

Orthogonal frequency division multiplexing (OFDM) [18] and multi-band carrierless amplitude and phase modulation (MB-CAP) [19] have been proposed as good candidates to upgrade inefficient modulation formats like on-off keying (OOK) or impulse radio (IR). Although both OFDM and MB-
CAP have compared similarly in dynamic and changing wireless scenarios, MB-CAP has shown better performance than MB-OFDM in wireless systems supported by a RoF-based fronthaul, achieving large capacities even under difficult wireless channel conditions [20]-[27]. CAP, which shares many similarities with quadrature amplitude modulation (QAM), uses two orthogonal signals to transmit data. Unlike QAM, where a local oscillator is required, CAP uses pulse shaping filters to generate the time-domain orthogonality [28], enabling less complex transceivers [29].

Benefits of MB-CAP regarding OFDM are various. Firstly, MB-CAP does not require electrical complex-to-real-value conversion which involves a complex mixer and radio frequency (RF) source for base-band signal modulation. Secondly, MB-CAP generally uses shaping filters based on the square-root raised cosine (SRRC) [19], which is a Nyquist filter. The digital signal processing (DSP) complexity of the MB-CAP transmitter system can drastically be reduced by the use of a look-up table [30], where the transmitted pulses are stored. On the other hand, SRRCs used in MB-CAP are finite impulse response (FIR) filters. These filters can be implemented in both analog or digital domains. The analog domain is preferred to reduce the system complexity for high number of bands $[19,30]$, while the digital domain is preferred due to low cost, easy reconfigurability, integrability and programmability [31]. Finally, MB-CAP shows higher power efficiency than OFDM [32] since the peak-to-average power ratio (PAPR) of OFDM is very high. Due to this fact, OFDM needs highly linear amplifiers, increasing the system cost.

Since MB-CAP transfers many of the operations to the digital domain, it provides a smooth overlapping with emerging paradigms beyond C-RAN, where functions will be splitted among the central unit $(\mathrm{CU})$ or $\mathrm{BBU}$, the radio unit (RU) or RRH and the distributed unit (DU), located between the CU and RU to fulfill strict requirements of future services in terms of bit rate and latency [33, 34]. Particularly, virtualized-RAN (VRAN), where many functions are digitized, the flexible design of MB-CAP signal parameters will play an important role to achieve the whole network potential [35]. This in turns opens the door to implement function accelerations in hardware,

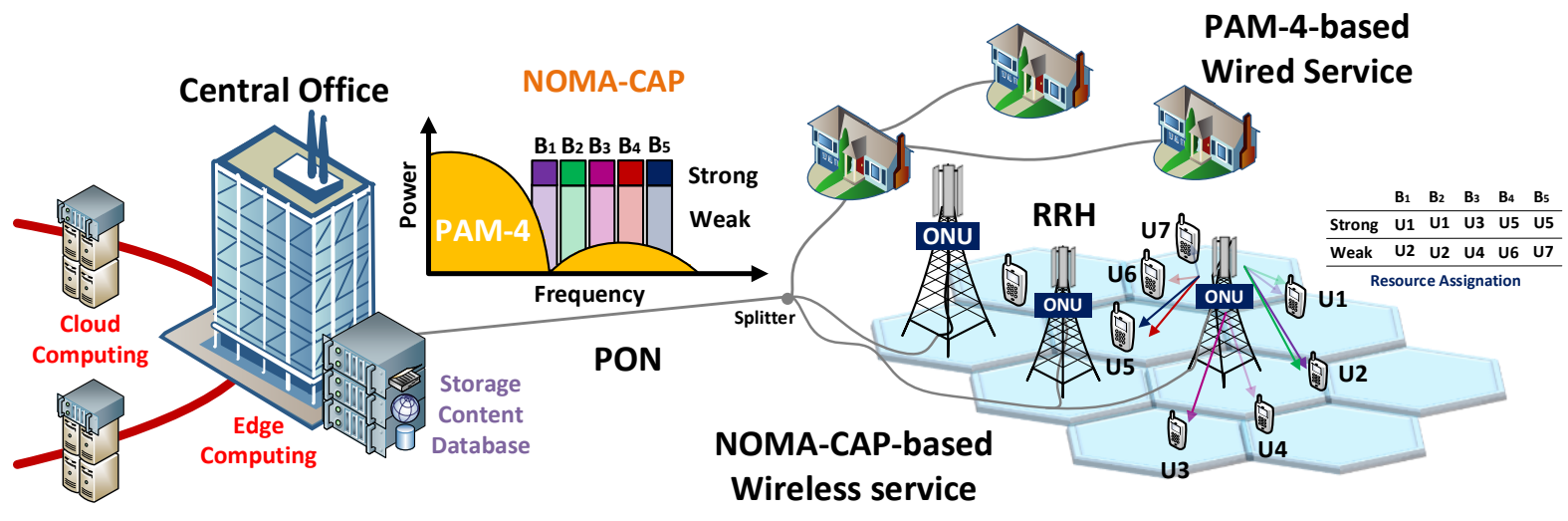

Fig. 1. Conceptual diagram of a converged fronthaul and passive optical network (PON). PAM-4 modulation format is used as legacy system and NOMA-CAP is used for future 5G+ fronthaul. Two NOMA levels (strong and weak) are considered per each CAP band. 

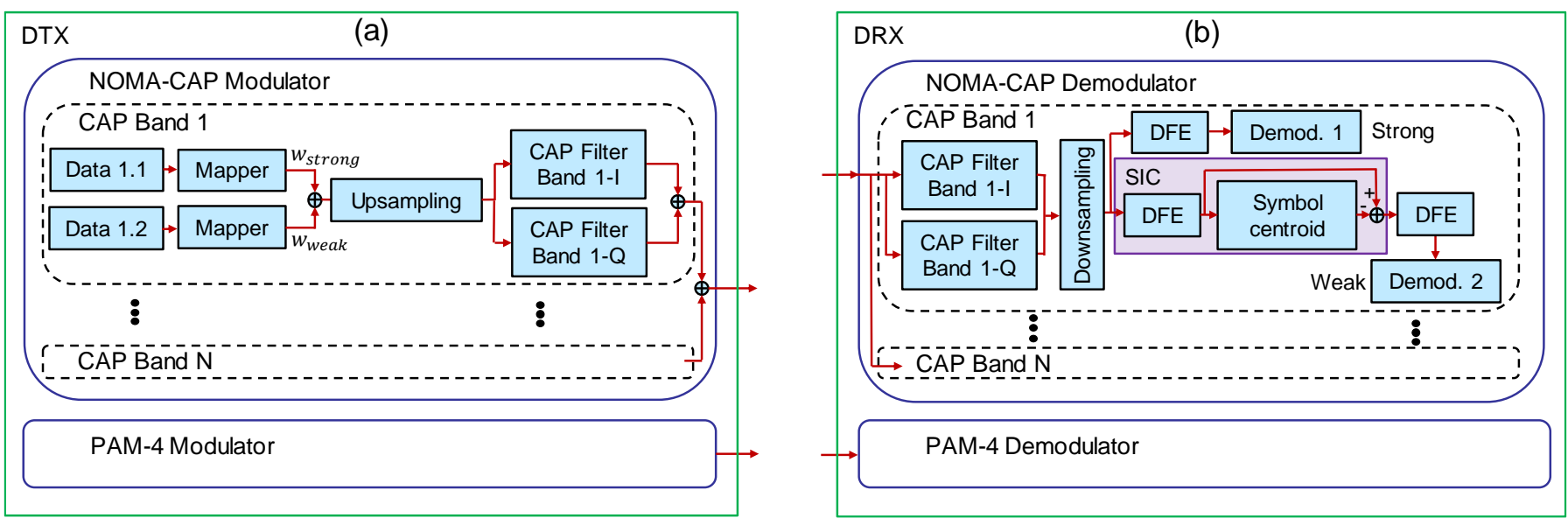

Fig. 2. DSP architecture of the converged fronthaul and fixed access system using NOMA-CAP and PAM-4: (a) the digital transmitter (DTX) and (b) digital receiver (DRX).

thereby improving the network performance [36].

NOMA and MB-CAP working in combination have been experimentally demonstrated in [21] as a viable choice for wireless multi-user provisioning. Although that work focused on the wireless domain, NOMA-CAP should also be investigated for optical applications not only as an appropriate modulation format for fronthaul transmission but also for its potential coexistence with other legacy modulation formats in converged wireless-wired network solutions. Recently, NOMA-CAP have been reported in [37]-[39] for wired services in PONs. However, the convergence of NOMA-CAP as a wireless signal in a converged fronthaul and fixed access system has only been studied with little detail in [40]. In this work, using RoF technology, we demonstrate the downlink transmission of a wireless NOMA-CAP waveform with two power levels per band multiplexed with a legacy single-carrier wired signal in a 5G+ PON scenario as shown in Fig. 1. Specifically, we experimentally assess a wireless-wired converged PON system which utilizes a $10 \mathrm{~Gb} / \mathrm{s}$ PAM-4 signal as the wired service. Thanks to its compatibility with current, cost-effective intensity modulation-direct detection (IM-DD) deployments and low complexity [41, 42], PAM-4 is considered the most straightforward modulation scheme for future optical access networks as an upgrade to NRZ-OOK. Two converged system implementations have been considered, the first one using electrical frequency division multiplying (EFDM) and the second one using hybrid EFDM-wavelength division multiplexing (EFDM-WDM). While the EFDM technology increases the system spectral efficiency, hybrid EFDM-WDM technology aims to increase the network capacity with a reasonable increase of complexity [43]. In both cases, the converged system assessment has been performed in terms of bit-error rate (BER) to determine the potential transmission penalties due to the signal interference and the fiber chromatic dispersion (CD). The results show the successful transmission of the converged wired and wireless signals providing a transmission bit rate of $10 \mathrm{~Gb} / \mathrm{s}$ and $15 \mathrm{~Gb} / \mathrm{s}$ for PAM-4 and NOMA-CAP signals, respectively.

The rest of the paper is organized as follows. Section II explains the digital signal processing (DSP) algorithms for generation/detection of NOMA-CAP with two power levels, the generation/detection procedure of PAM-4 signals, the experimental optical transmission setup and the NOMA power ratio characterization. Section III shows and discusses the measured results for NOMA-CAP and PAM-4 in the proposed converged PON scenarios, with Section IV concluding and summarizing the manuscript.

\section{DESCRIPTION OF THE EXPERIMENTAL SETUP}

\section{A. NOMA-CAP and PAM-4 generation, detection and digital} signal processing (DSP) algorithms

Fig. 2(a) shows the transmitter DSP architecture for the converged mobile fronthaul and fixed access system using NOMA-CAP and PAM-4 signals. Two NOMA levels per band, corresponding two different users (see Fig. 1), were considered for the NOMA-CAP signal. In each band of the NOMA-CAP signal, the data of the two levels were independently mapped to four-level quadrature amplitude modulation (4-QAM) symbols. The two NOMA levels, which are referred as "strong NOMA" and "weak NOMA", were power weighted according to the power ratio $r_{p}=20 \log \left(w_{\text {strong }} / w_{\text {weak }}\right)$, where $w_{\text {strong }}$ and $w_{\text {weak }}$ are the weighs of the strong and weak NOMA levels, respectively. Then, both signals were added and its sample rate increased to the sampling frequency-symbol rate ratio per band. To enable a bit rate of $1 \mathrm{~Gb} / \mathrm{s}$ per band, i.e., $0.5 \mathrm{~Gb} / \mathrm{s}$ per NOMA level, the baud rate was fixed to $0.25 \mathrm{GBd}$. Next, the real and imaginary parts of the complex NOMA signal were separated and independently filtered with the pair of CAP orthogonal filters, which form a Hilbert transform pair, for a specific band. Eq. (1) and Eq. (2) define, in the time domain, the orthogonal filters used for each band, $h_{I_{i}}$ and $h_{Q_{i}}$, being $p(t)$ and $f_{c_{i}}$ the pulse shaping filter and the carrier sub-frequency of the CAP band $i$, respectively. A square-root raised cosine (SRRC) filter with a roll-off factor of 0.05 and 20 symbols was employed as $p(t)$ to optimize the spectral efficiency of the CAP signal. Therefore, since the SRRC matched filters of each CAP band are used at the reception side, the signals can be extracted and the inter-symbol interference (ISI) is simultaneously minimized. Finally, the NOMA-CAP bands were added to generate the complete NOMA-CAP signal to be transmitted 

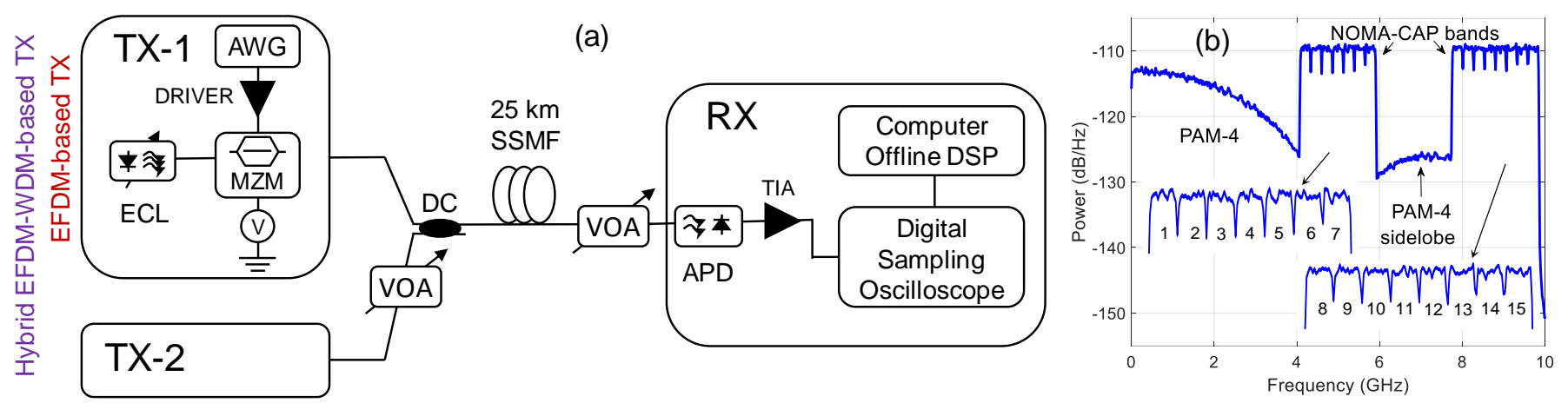

Fig. 3. (a) Experimental PON setup. (Acronyms) ECL: external cavity laser, V: voltage source, AWG: arbitrary waveform generator, DC: directional coupler, VOA: variable optical attenuator, APD: avalanche photodiode, TIA: transimpedance amplifier. (b) Example of measured spectra of converged NOMA-CAP and PAM-4 services, providing a total bit rate of $25 \mathrm{~Gb} / \mathrm{s}$ in just $10 \mathrm{GHz}$ of transmission bandwidth.

$y_{\text {NOMA-CAP. }}$ The PAM modulator was configured to work with a uniform four-level constellation, generating the baseband PAM-4 signal to transmit $y_{P A M-4}$. For the EFDM implementation, the NOMA-CAP and the PAM-4 signals were digitally added to form the full data transmitted signal. Note that, since the baud rate of both PAM-4 (5 GBd) and NOMACAP $(0.25 \mathrm{GBd})$ signals are different, the PAM-4 signal has to be upsampled before their digital addition.

$$
\begin{aligned}
& h_{I_{i}}=p(t) \cos \left(2 \pi f_{c_{i}} t\right) \\
& h_{Q_{i}}=p(t) \sin \left(2 \pi f_{c_{i}} t\right)
\end{aligned}
$$

Fig. 2(b) shows the receiver DSP architecture for both NOMA-CAP and PAM-4 signals. Firstly, each MB-CAP band was filtered using the inverse of the orthogonal filters used at the transmitter for each CAP band. The strong NOMA level DSP required a decision feedback equalizer (DFE) with 30 forward and 20 backward taps, followed by the de-mapping process. The weak NOMA level reception required additional DSP steps like SIC, as shown in Fig. 2(b). This consisted of a DFE, the calculation of the symbol centroid of the strong NOMA level and subtraction from the DFE-filtered signal. After the SIC process, a DFE was applied again to the resulting signal and finally the signal was de-mapped. The parameters of the DFEs for the weak NOMA level were the same as the DFE used for the strong NOMA level. In the case of the PAM demodulator, the received signal was optimally filtered using a finite impulse response (FIR) low-pass filter with a cut-off frequency of eighty percent of the PAM symbol rate. A 13-tapFIR-based adaptive equalizer was performed to achieve the maximum system performance at low receiver complexity. A higher number of taps does not result in any significant improvement. The tap weights were updated with a decisiondirected least-mean square (DD-LMS) algorithm [44].

\section{B. Converged PON experimental setup}

Fig. 3(a) shows the experimental IM-DD-based setup. The optical transmitter, named TX-1 in the figure, used an external cavity laser (ECL) tuned at $1548 \mathrm{~nm}$ and external modulation with a Mach-Zehnder modulator (MZM) biased at its quadrature point. An arbitrary waveform generator (AWG) with a $3 \mathrm{~dB} 20 \mathrm{GHz}$ electrical bandwidth and set at $20 \mathrm{GSa} / \mathrm{s}$ was used to drive the modulator with the aggregated NOMACAP/PAM-4 signal in the EFDM-based converged system. The electrical driver output power was chosen to ensure the MZI linear regimen, maximizing the adjacent channel power leakage ratio (ACLR), and hence increasing the spectral efficiency of the system [45]-[47]. The transmitter output power was $+3 \mathrm{dBm}$. After modulation, the optical signal was launched into a $25 \mathrm{~km}$ span of standard single-mode fiber (SSMF). The optical receiver (RX) was based on an avalanche photodiode (APD) followed by a transimpedance amplifier (TIA). The APD dynamic range was of $20 \mathrm{~dB}$. The received optical power
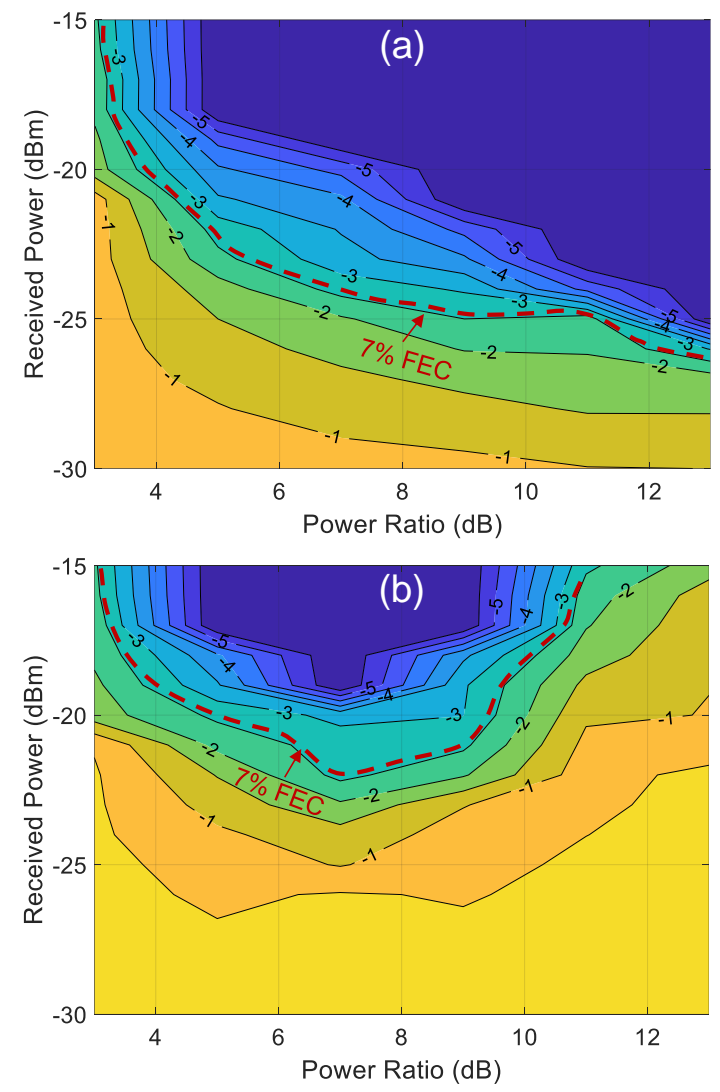

Fig. 4. Contour $\log _{10}$ (BER) vs. received power vs. power ratio plots in backto-back configuration for: (a) strong NOMA and (b) weak NOMA levels. 
before the APD was set with a variable optical attenuator (VOA) to study the system performance in terms of the receiver sensitivity. In this work, only one receiver was considered for simplicity but the different power multiplexed signals and the PAM-4 signal can be detected by different receivers as shown in Fig. 1. An additional transmitter, named TX-2 and with similar characteristics to TX-1, was added to the setup to evaluate the converged system performance using the hybrid EFDM-WDM scenario. In this case, the second laser was tuned at $1548.2 \mathrm{~nm}$ to minimize both the optical beat interference due to unwanted mixing products generated during the direct detection process at the receiver [48]-[50] and the optical gap between both lasers. Note that, for the hybrid implementation, although the NOMA-CAP and PAM-4 signals are independently generated by two different transmitters, the EFDM technique is conducted as well to avoid the complete overlap of both signals in the frequency domain in the detection process. A second VOA was added to the TX-2's output to control the optical power of the interfering signal. Finally, the detected signal was digitalized with a sampling frequency of $25 \mathrm{GSa} / \mathrm{s}$ using a real-time oscilloscope and then processed offline. Fig. 3(b) shows the composite electrical spectrum measured at the receiver side, showing the NOMA-CAP signal with fifteen $0.25 \mathrm{GHz} \mathrm{CAP}$ bands and providing a total aggregated bit rate of $15 \mathrm{~Gb} / \mathrm{s}$. The NOMA-CAP bands numbered 1 to 7 were placed in the spectral null between the main lobe and the sidelobe of the $10 \mathrm{~Gb} / \mathrm{s}$ PAM-4 signal, while additional NOMA-CAP bands numbered 8 to 15 were accommodated within the frequency range between $8 \mathrm{GHz}$ and $10 \mathrm{GHz}$. Those locations were chosen to minimize the crosstalk interference between the PAM-4 and NOMA-CAP signals, as it shall be shown in the next Section.

\section{Experimental NOMA power characterization}

NOMA can maintain user-fairness and diverse quality of service by flexible power control between the strong and weak users; particularly, as more power is allocated to a weak user, NOMA offers, for example, higher cell-edge throughput and thus enhances the cell-edge user experience. In a C-RAN scenario as shown in Fig. 1, where RoF-based fronthaul is considered, the user power assignation must be performed in the BBU located at the CO. Electro-optical conversion is required to transmit the users signal from the BBU to the RRH. Therefore, the characterization of NOMA power ratio variation at the optical domain is required. Fig. 4 shows the BER vs. $r_{p}$ vs. the received optical power $P_{R X}$ for the weak and strong NOMA levels for the case when a single CAP band of $0.25 \mathrm{GHz}$ is transmitted in back-to-back. For the strong NOMA level in Fig. 4(a), the BER performance gradually increases as $P_{R X}$ increases. This is because higher values of $P_{R X}$ increase the optical signal power hence yielding a better signal-to-noise ratio (SNR). Furthermore, when $r_{p}$ increases, the performance (a)

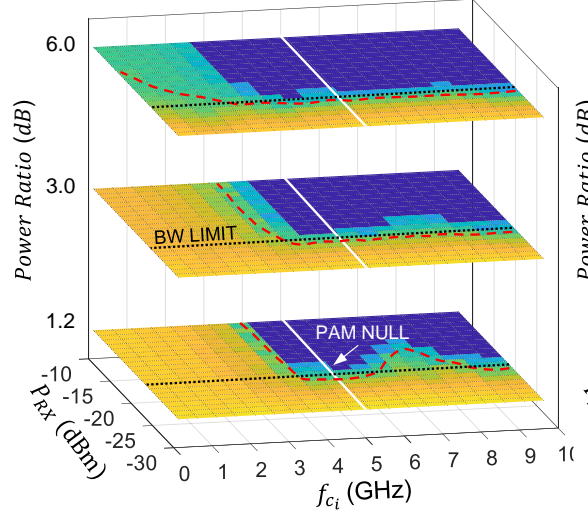

(b)

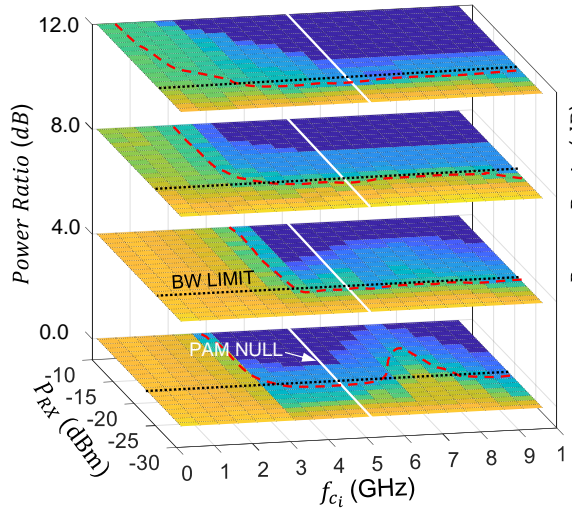

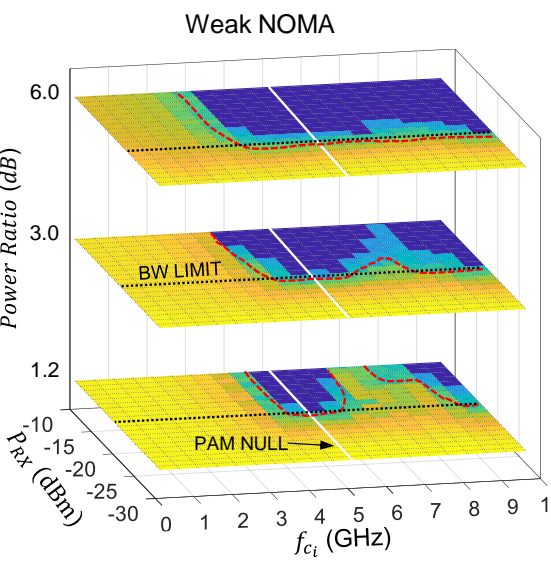

Weak NOMA

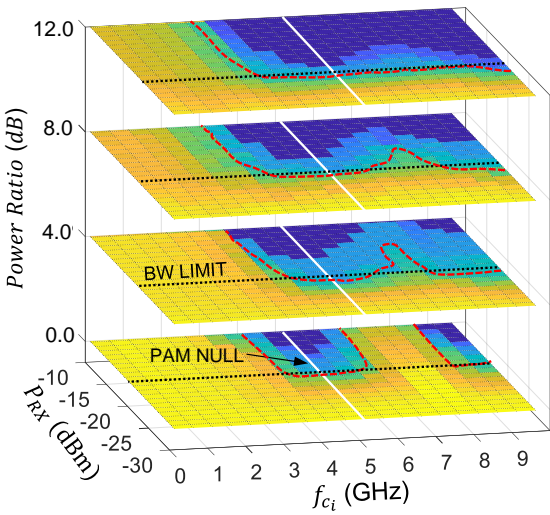

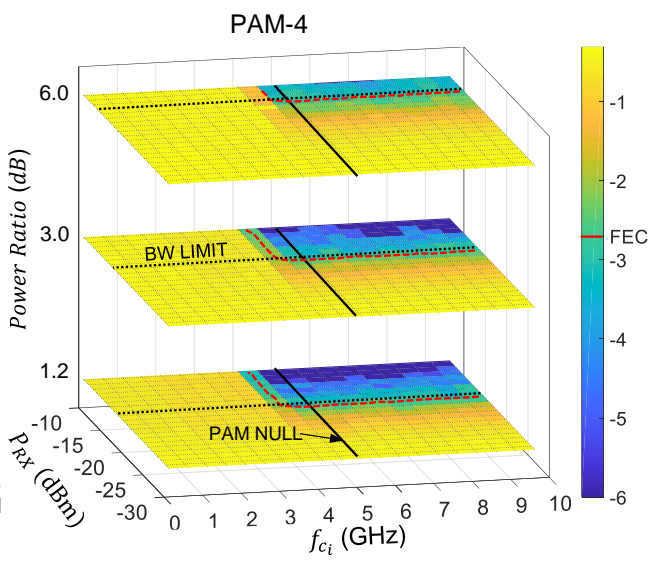

PAM-4

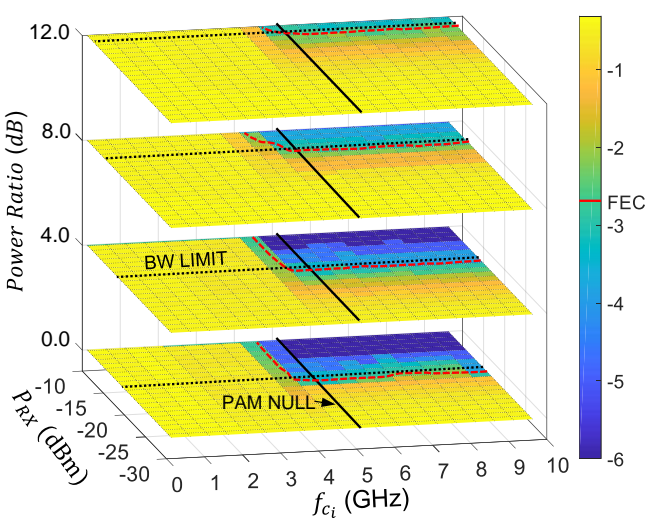

Fig. 5. Contour $\log _{10}(\mathrm{BER})$ vs. received power vs. frequency carrier $\left(f_{c_{i}}\right)$ of a NOMA-CAP signal with just one $0.25 \mathrm{GHz}$ CAP band for the weak and strong NOMA levels and the $10 \mathrm{~Gb} / \mathrm{s}$ in back-to-back configuration when the NOMA-CAP and PAM-4 signals are generated using: (a) EFDM technology and (b) hybrid EFDM-WDM technology. Dash red line is the 7\% FEC limit. 
TABLE I

SUMMARY OF RECEIVER SENSITIVITIES $(S S)$ AND RANGE OF $f_{c_{i}}$ IN WHICH THE PENALTY IS LESS THAN $1 \mathrm{DB}\left(\Gamma=R_{f_{c_{i}} \mid \Delta S \leq 1 d B}\right)$ OF THE TWO NOMA-CAP LEVEL SIGNALS AND PAM-4, AS WELL AS THE EFFECTIVE BANDWIDTH OF THE SYSTEM FOR THE WIRELESS TRANSMISSION $\left(B W_{\text {eff }}\right)$ OF THE CONVERGED SYSTEM USING EFDM TECHNOLOGY.

\begin{tabular}{|c|c|c|c|c|c|c|}
\hline \multirow{2}{*}{$\frac{\text { Power Ratio }(\mathrm{dB})}{\text { Parameter }}$} & \multicolumn{2}{|r|}{1.2} & \multicolumn{2}{|r|}{3.0} & \multicolumn{2}{|c|}{6.0} \\
\hline & $S(\mathrm{dBm})$ & $\Gamma(\mathrm{GHz})$ & $S(\mathrm{dBm})$ & $\Gamma(\mathrm{GHz})$ & $S(\mathrm{dBm})$ & $\Gamma(\mathrm{GHz})$ \\
\hline $\begin{array}{c}\text { Strong } \\
\text { NOMA-CAP }\end{array}$ & -22.2 & {$[3.8,6.2],[8.2,10]$} & -23.1 & {$[3.6,10]$} & -23.2 & {$[2.1,10]$} \\
\hline PAM-4 & -18.2 & {$[4.1,10]$} & -17.2 & {$[4.3,10]$} & -13.4 & {$[4.5,10]$} \\
\hline
\end{tabular}

TABLE II

SUMMARY OF RECEIVER SENSITIVITIES $(S \mathrm{~S})$ AND RANGE OF $f_{c_{i}}$ IN WHICH THE PENALTY IS LESS THAN 1 DB $\left(\Gamma=R_{f_{c_{i}} \mid \Delta S \leq 1 d B}\right)$ OF THE TWO NOMA-CAP LEVEL SIGNALS AND PAM-4, AS WELL AS THE EFFECTIVE BANDWIDTH OF THE SYSTEM FOR THE WIRELESS TRANSMISSION $\left(B W_{e f f}\right)$ OF THE CONVERGED SYSTEM USING HYBRID EFDM-WDM TECHNOLOGY.

\begin{tabular}{|c|c|c|c|c|c|c|c|c|}
\hline \multirow{2}{*}{$\begin{array}{c}\text { Power Ratio }(\mathrm{dB}) \\
\text { Parameter }\end{array}$} & \multicolumn{2}{|r|}{0.0} & \multicolumn{2}{|r|}{4.0} & \multicolumn{2}{|r|}{8.0} & \multicolumn{2}{|c|}{12.0} \\
\hline & $S(\mathrm{dBm})$ & $\Gamma(\mathrm{GHz})$ & $S(\mathrm{dBm})$ & $\Gamma(\mathrm{GHz})$ & $S(\mathrm{dBm})$ & $\Gamma(\mathrm{GHz})$ & $S(\mathrm{dBm})$ & $\Gamma(\mathrm{GHz})$ \\
\hline $\begin{array}{c}\text { Strong } \\
\text { NOMA-CAP }\end{array}$ & -23.5 & {$[3.5,6.2],[8.3,10.0]$} & -24.2 & {$[3.5,10.0]$} & -25.3 & {$[3.0,10.0]$} & -26.1 & {$[2.2,10]$} \\
\hline $\begin{array}{c}\text { Weak } \\
\text { NOMA-CAP }\end{array}$ & -20.4 & {$[4.1,5.9],[9.0,10.0]$} & -21.8 & {$[4.0,6.2],[7.7,10.0]$} & -22.6 & {$[3.6,6.0],[6.9,10.0]$} & -23.7 & {$[3.1,10]$} \\
\hline PAM-4 & -18.5 & {$[4.4,10.0]$} & -17.8 & {$[4.3,10.0]$} & -14.3 & {$[4.6,10.0]$} & -11.7 & {$[4.8,10.0]$} \\
\hline
\end{tabular}

in terms of BER raises since the SNR of the strong NOMA level is higher, resulting in a better receiver sensitivity, defined as the minimal $P_{R X}$ to achieve a BER threshold of 2.2E-3, corresponding to a $7 \%$ forward error corrections (FEC) overhead [51]. Hence, higher $r_{p}$ values are preferred for the strong NOMA level because it reduces the interference of the weak NOMA level, as it can be observed in Fig. 4(a). Similarly, the BER performance for the weak NOMA level also improves as $P_{R X}$ increases as shown in Fig. 4(b). Nevertheless, the BER performance of the weak NOMA level decreases for both lower and higher $r_{p}$ values. This is due to the error propagation from the strong NOMA level decoding caused by SIC cancellation in the demodulation process of the weak NOMA level signal. Therefore, the weak NOMA level signal needs a high $P_{R X}$ value and an intermediate $r_{p}$ value as shown in Fig. 4(b). As a good trade-off, a value of $r_{p}=7 \mathrm{~dB}$ was chosen to optimize the reception of both NOMA levels. Similar behavior was obtained after transmission over $25 \mathrm{~km}$ of SSMF.

\section{EXPERIMENTAL RESULTS AND DISCUSSION}

In a converged PON scenario like the one shown in Fig. 1, where wired and wireless services can be delivered over a single fiber, the key target is to optimize the best spectral allocation for the wireless signal that minimizes the mutual crosstalk interference of the co-propagated wired-wireless services and simultaneously maximizes the transmission bandwidth utilization of the system. Figs. 5(a) and 5(b) show, respectively, the converged system crosstalk performance measured in terms of the BER vs. $P_{R X}$ vs. $f_{c_{i}}$ of a NOMA-CAP signal with just one
CAP band of $0.25 \mathrm{GHz}$ when the NOMA-CAP and PAM-4 signals are multiplexed in the electrical domain and generated using the same transmitter (EFDM implementation) and when the two signals are generated using separated transmitters with different transmission wavelengths and performing EFDM (hybrid EFDM-WDM implementation). In all these measurements, $f_{c_{i}}$ was swept form $0 \mathrm{GHz}$ to $10 \mathrm{GHz}$ in steps of $125 \mathrm{MHz}$ and the $r_{p}$ among the two NOMA levels was fixed to the optimized value of $7 \mathrm{~dB}$ found in the previous section. To study the effect of the crosstalk interference, Fig. 5 shows the BER curves as a function of the power ratio $(P R)$ between the NOMA-CAP and PAM-4 signals. Note that, for the EFDM implementation, this power ratio is set digitally, i.e., through the variation of the amplitude of the PAM-4 signal samples, while in the hybrid EFDM-WDM implementation, the $P R$ is controlled optically through the variation of the optical power of the PAM-4 signal using a VOA at the transmitter output (see Fig. 3).Table I and Table II summarize the receiver sensitivities $(S s)$ of the weak and strong NOMA levels and the $10 \mathrm{~Gb} / \mathrm{s}$ PAM-4 signal when the NOMA-CAP signal is centered in the null point of the PAM-4 signal, at $5 \mathrm{GHz}$, as well as the ranges of $f_{c_{i}}$ in which the penalty in the receiver sensitivity $(\Delta S)$ due to the crosstalk is lower than $1 \mathrm{~dB}\left(R_{\left.f_{c_{i}}\right|_{\Delta S \leq 1 d B}}\right)$ as shown in Figs. 5(a) and 5(b) for different values of the $P R$ between the NOMA-CAP and PAM-4 signals. Finally, Table I and Table II also show the effective bandwidth $\left(B W_{e f f}\right)$ range and the total $B W_{\text {eff }}$ resulting to be used by the NOMA-CAP transmission in each considered converged system implementation. Specifically, the $B W_{\text {eff }}$ range is defined as the intersection 

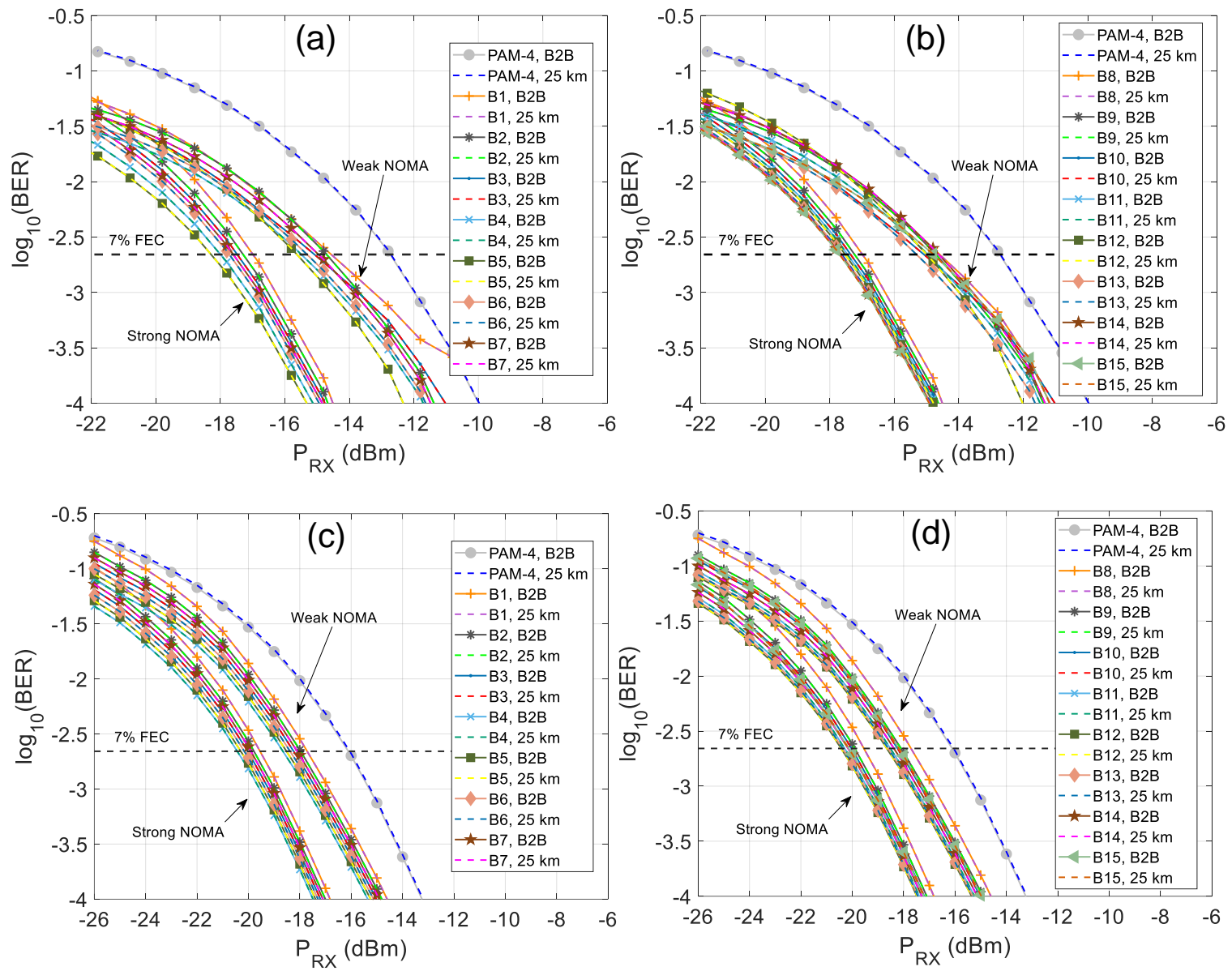

Figure 6. BER vs. received power $\left(P_{R X}\right)$ of $10 \mathrm{~Gb} / \mathrm{s}$ PAM-4 and of each CAP band of a NOMA-CAP signal with two NOMA levels and 15 CAP bands in backto-back (B2B) configuration and over $25 \mathrm{~km}$ of SSMF for: (a) the NOMA-CAP bands with ID form 1 to 7 and, (b) the NOMA-CAP bands with ID form 8 to 15 with the EFDM system and, (c) the NOMA-CAP bands with ID form 1 to 7 and, (d) the NOMA-CAP bands with ID form 8 to 15 with the hybrid EFDM-WDM system.

between $R_{f_{c_{i}} \Delta S \leq 1 d B}$ of the two NOMA signals and the PAM-4 for a specific $P R$ value, while total $B W_{\text {eff }}$ is transmission bandwidth available for the NOMA-CAP signal. In other words, $B W_{\text {eff }}$ is the bandwidth utilized by the NOMA-CAP overlapped signal.

As it can be observed in Table 1, the higher the $P R$, the higher the available transmission bandwidth for the NOMA-CAP signal is. This is because for higher $P R$ values, the PAM-4 signal power is lower and hence decreasing the interference over the NOMA-CAP signal. Note that for $P R=\{1.2,3.0\} \mathrm{dB}$, the frequency range to accommodate the wireless signal is discontinuous due to the higher energy of the PAM-4 side-lobe. In the case of the $10 \mathrm{~Gb} / \mathrm{s}$ PAM- 4 , decreasing the $P R$ ratio between the NOMA-CAP and PAM-4 signals corresponds to an improvement of the PAM-4 receiver sensitivity, from $-13.4 \mathrm{dBm}$ for $P R=6 \mathrm{~dB}$ to $-18.2 \mathrm{dBm}$ for $P R=1.2 \mathrm{~dB}$. From Table II, similar $B W_{\text {eff }}$ behavior can be observed for the converged system based on the hybrid EFDM-WDM implementation. Thus, for $P R=12.0 \mathrm{~dB}$, the crosstalk interference of the PAM-4 signal is low, consequently leading to higher receiver sensitivities for the two NOMA-CAP levels and increasing $B W_{\text {eff }}$ up to $5.2 \mathrm{GHz}$. Nevertheless, the PAM-4 receiver sensitivity is only $-11.4 \mathrm{dBm}$. On the contrary, for
$P R=0.0 \mathrm{~dB}$, the receiver sensitivity for PAM-4 is of $-18.5 \mathrm{dBm}$, and the crosstalk over the NOMA-CAP signal is higher, deteriorating the NOMA-CAP receiver sensitivities and reducing $B W_{\text {eff }}$ to $2.5 \mathrm{GHz}$. As a trade-off solution, we consider $R P=3 \mathrm{~dB}$ and $P R=4 \mathrm{~dB}$ for the converged system implementation based on the EFDM and hybrid EFDM-WDM technologies, respectively, both implementations providing and effective bandwidth close to $4.2 \mathrm{GHz}$ where fifteen NOMACAP bands of $0.25 \mathrm{GHz}$ can be accommodated as shown in Fig. 3(b).

Finally, for the back-to-back configuration and $25 \mathrm{~km}$ of SSMF transmission, Figs. 6(a, b, c, d) show the BER vs. $P_{R X}$ curves for the fifteen NOMA-CAP bands, providing a total aggregated bit rate of $15 \mathrm{~Gb} / \mathrm{s}$ and the $10 \mathrm{~Gb} / \mathrm{s}$ PAM- 4 , are transmitted simultaneously using EFDM and hybrid EFDMWDM technologies and considering $P R=3.0 \mathrm{~dB}$ and $P R=$ $4 \mathrm{~dB}$, respectively. From Figs. 6(a, b), the receiver sensitivities of the converged system based on EFDM technology for the strong and weak NOMA levels are, averaged for all bands, $-17.2 \mathrm{dBm},-15.3 \mathrm{dBm}$ respectively, and for $10 \mathrm{~Gb} / \mathrm{s}$ PAM-4, the receiver sensitivity is $-12.8 \mathrm{dBm}$. In the case in which the converged system is based on hybrid EFDM-WDM technology, the receiver sensitivities for the strong NOMA, weak NOMA and PAM-4 signals are $-18.3 \mathrm{dBm},-20.1 \mathrm{dBm}$ 
and $-16.1 \mathrm{dBm}$, respectively. Better receiver sensitivities can be achieved through a cost-effective quasi-coherent receiver [11, 52], potentially providing a receiver sensitivity enhancement of 14 dB. From Figs. 6(a, b, c, d), no transmission penalty due to $\mathrm{CD}$ or any other impairment was observed in the receiver sensitivities after $25 \mathrm{~km}$ transmission. The better performance of the NOMA-CAP signals shown in Fig. 6 is due to the fact the high tolerance of the multiband-CAP (MB-CAP) signals to the transmission system impairments. On the one hand, the bandwidth of each CAP band is 20 times lower than the $10 \mathrm{~Gb} / \mathrm{s}$ PAM-4 signal, leading to a higher tolerance to bandwidth system limitations. On the other hand, as demonstrated in [19], for lower MB-CAP band bandwidth values, the system CD tolerance increases notably for short-haul transmission distances as considered in this paper, and hence there is no need for CD compensation.

\section{CONCLUSION}

The convergence of NOMA-CAP, considered as a novel 5G+ candidate modulation format, with PAM-4 within a singlecarrier wired service has been experimentally demonstrated in a wireless-wired converged PON scenario. The transmission of fifteen NOMA-CAP bands, providing a total aggregated bit rate of $15 \mathrm{~Gb} / \mathrm{s}$ and $10 \mathrm{~Gb} / \mathrm{s}$ PAM-4, over $25 \mathrm{~km}$ of SSMF has been investigated with negligible BER penalty for two different converged system implementations, the first using EFDM and the second using EFDM-WDM. In the first case, the NOMACAP bands were aggregated and digitally added to the baseband PAM-4 waveform to form the whole data transmitted signal. To evaluate the crosstalk interference between the NOMA-CAP and PAM-4 signals, the PAM-4 modulator output was amplitude-weighted with different values. In the second case, the NOMA-CAP and PAM-4 signals were generated with two different transmitters and the optical crosstalk due to the variation of the optical power difference between both signals was generated with a variable optical attenuator. Both system implementations yield the same effective bandwidth for the wireless signal of $4.2 \mathrm{GHz}$ in terms of the receiver sensitivity, however the hybrid EFDM-WDM technology shows a $3 \mathrm{~dB}$ sensitivity gain compared to the EFDM technology.

The proposed convergence of a $10 \mathrm{~Gb} / \mathrm{s}$ PAM- 4 wired signal with a $15 \mathrm{~Gb} / \mathrm{s}$ NOMA-CAP wireless waveform, with a potential increase in the number of wavelength channels in future WDM access networks, could be a good choice for future augmentation of currently deployed PON networks to meet the efficient advancement of future $5 \mathrm{G}+$ fronthaul networks.

\section{REFERENCES}

[1] Cisco, "Cisco visual networking index: Global mobile data traffic forecast update, 2016-2021 white paper," Cisco, Jialefuniya, America, Tech. Rep. Cisco white paper, 2017.

[2] A. Martinez, et al., "Simultaneous baseband and RF optical modulation scheme for feeding wireless and wireline heterogeneous access networks," IEEE Trans. Microwave Theory Tech., vol. 49, no. 10, pp. 2018-2024, Oct. 2001.

[3] J.J. Vegas Olmos, X. Pang, A. Lebedev, M. Sales, I. Tafur Monroy, "Wireless and wireline service convergence in next generation optical access networks - the FP7 WISCON project," IEICE Transactions on Communications, E97-B, pp. 1537-1546, 2014.

[4] AG. Sun, K. Xiong, G. O. Boateng, D. Ayepah-Mensah, G. Liu and W. Jiang, "Autonomous Resource Provisioning and Resource Customization for Mixed Traffics in Virtualized Radio Access Network," IEEE Systems Journal, vol. 13, no. 3, pp. 2454-2465, 2019.

[5] P. Iovanna, F. Cavaliere, S. Stracca, L. Giorgi and F. Ubaldi, "5G Xhaul and service convergence: transmission, switching and automation enabling technologies," IEEE Journal of Lightwave Technology, Early Access.

[6] F. Dominique, "Requirements of 5G Radio Networks on Optical X-haul Transport," in Proc. 2019 Optical Fiber Communication Conference (OFC), San Diego, CA, pp. 1-3, 2019.

[7] e-CPRI, http://www.cpri.info/spec.html.

[8] H. Kim, "RoF-based Optical Fronthaul Technology for 5G and Beyond," in Proc. 2018 Optical Fiber Communications Conference and Exposition (OFC), San Diego, CA, pp. 1-3, 2018.

[9] B. G. Kim, H. Kim, and Y. C. Chung, "Impact of multipath interference in the performance of RoF-based mobile fronthaul network implemented by using DML," Journal of Lightwave Technology, vol. 35, pp.145-151, 2017.

[10] B. G. Kim, S. H. Bae, H. Kim and Y. C. Chung, "Feasibility of RoF-based optical fronthaul network for next-generation mobile communications," in Proc. 2017 Opto-Electronics and Communications Conference (OECC) and Photonics Global Conference (PGC), Singapore, 2017, pp. 1-2.

[11] S. Sarmiento, J. A. Altabas, S. Spadaro and J. A. Lazaro, "Experimental Assessment of 10 Gbps 5G Multicarrier Waveforms for High-Layer Split U-DWDM-PON-Based Fronthaul," IEEE Journal of Lightwave Technology, vol. 37, no. 10, pp. 2344-2351, 2019.

[12] S. Dang, O. Amin, B. Shihada and M. Alouini, "What should 6G be?," Nature Electronic, vol. 3, pp. 20-29, 2020.

[13] L. Dai et al., "Non-orthogonal multiple access for 5G: solutions, challenges, opportunities, and future research trends," IEEE Commun. Mag., vol. 53, no. 9, pp. 74-81, 2015.

[14] H. Kenichi and B. Anass, "Non-orthogonal multiple access (NOMA) with successive interference cancellation for future radio access," IEICE Transactions Communications, vol. 98, no 3, p. 403-414, 2015.

[15] V. V. Veeravalli and A. Mantravadi, "The coding-spreading tradeoff in CDMA systems," IEEE Journal on Selected Areas in Communications, vol. 20, no. 2, pp. 396-408, 2002.

[16] M. Al-Imari, P. Xiao, M. A. Imran and R. Tafazolli, "Uplink nonorthogonal multiple access for 5G wireless networks," 11th International Symposium on Wireless Communications Systems (ISWCS), Barcelona, pp. 781-785, 2014.

[17] L. Dai, B. Wang, Y. Yuan, S. Han, C. I and Z. Wang, "Non-orthogonal multiple access for $5 \mathrm{G}$ : solutions, challenges, opportunities, and future research trends," IEEE Communications Magazine, vol. 53, no. 9, pp. 74$81,2015$.

[18] V. Sipal et al., "Adaptive OFDM for wireless interconnect in confined enclosures," IEEE Wireless Commun. Lett., vol. 2, no. 5, pp. 507-510, 2013.

[19] M. I. Olmedo et al., "Multiband carrierless amplitude phase modulation for high capacity optical data links," IEEE Journal of Lightwave Technology, vol. 32, no. 4, pp. 798-804, 2014.

[20] R. Puerta, S. Rommel, J. J. Vegas Olmos, and I. Tafur Monroy, “Optically generated single side-band radio-over-fiber transmission of $60 \mathrm{Gbit} / \mathrm{s}$ over 50m at W-band," 2017 Optical Fiber Communications Conference and Exhibition (OFC), Los Angeles, CA, pp. 1-3, 2017.

[21] J. A. Altabas et al., "Nonorthogonal Multiple Access and Carrierless Amplitude Phase Modulation for Flexible Multiuser Provisioning in 5G Mobile Networks," IEEE Journal of Lightwave Technology, vol. 35, no. 24, pp. 5456-5463, 2017.

[22] D. Li et al., "Multi-band carrierless amplitude and phase modulation in RoF system for enhanced reliable mobile fronthaul," 2018 Conference on Lasers and Electro-Optics (CLEO), San Jose, CA, 2018, pp. 1-2.

[23] Junwen Zhang, Jianjun $\mathrm{Yu}$, Nan $\mathrm{Chi}$, Fan $\mathrm{Li}$, and Xinying $\mathrm{Li}$, "Experimental demonstration of 24-Gb/s CAP-64QAM radio-over-fiber system over 40-GHz mm-wave fiber-wireless transmission," Opt. Express, vol. 21, no. 22, pp. 26888-26895, 2013.

[24] M. Xu et al., "Multiband OQAM CAP modulation in MMW RoF systems with enhanced spectral and computational efficiency", 2016 Optical Fiber Communications Conference and Exhibition (OFC), Anaheim, CA, 2016, pp. 1-3.

[25] R. Puerta, S. Rommel, J. J. V. Olmos and I. T. Monroy, "10Gb/s ultrawideband wireless transmission based on multi-band carrierless amplitude phase modulation," 2016 IEEE 17th Annual Wireless and Microwave Technology Conference (WAMICON), Clearwater, FL, pp. $1-4,2016$ 
[26] S. Rommel, R. Puerta, J. J. V. Olmos and I. T. Monroy, "Capacity enhancement for hybrid fiber-wireless channels with $46.8 \mathrm{Gbit} / \mathrm{s}$ wireless multi-CAP transmission over 50m at W-band," 2017 Optical Fiber Communications Conference and Exhibition (OFC), Los Angeles, CA, pp. 1-3, 2017.

[27] R. Puerta, S. Rommel, J. J. V. Olmos and I. T. Monroy, "Up to 35 Gbps ultra-wideband wireless data transmission links," 2016 IEEE 27th Annual International Symposium on Personal, Indoor, and Mobile Radio Communications (PIMRC), Valencia, pp. 1-5, 2016

[28] G. Im, D. B. Harman, G. Huang, A. V. Mandzik, M. Nguyen and J. Werner, "51.84 Mb/s 16-CAP ATM LAN standard," IEEE J. Sel. Areas Commun., vol. 13, no. 4, pp. 620-632, 1995.

[29] J. L. Wei et al., "Performance and Power Dissipation Comparisons Between $28 \mathrm{~Gb} / \mathrm{s}$ NRZ, PAM, CAP and Optical OFDM Systems for Data Communication Applications," IEEE Journal of Lightwave Technology, vol. 30, no. 20, pp. 3273-3280, 2012.

[30] J. L. Wei, C. Sanchez, and E. Giacoumidis, "Fair comparison of complexity between a multi-band CAP and DMT for data center interconnects," Opt. Lett., vol. 42, pp. 3860-3863, 2017.

[31] F. M. Wu et al., "Performance Comparison of OFDM Signal and CAP Signal Over High Capacity RGB-LED-Based WDM Visible Light Communication," IEEE Photonics Journal, vol. 5, no. 4, pp. 79015077901507, Aug. 2013, Art no. 7901507.

[32] P.A. Haigh, A. Burton, K. Werfli, H. Le Minh, E. Bentley, P. Chvojka, W.O. Popoola, I. Papakonstantinou, S. Zvanovec, "A multi-CAP visiblelight communications system with $4.85-\mathrm{b} / \mathrm{s} / \mathrm{Hz}$ spectral efficiency", IEEE J. Sel. Areas Commun., vol 33 pp. 1771-1779, 2015.

[33] F. Giannone et al., "Impact of Virtualization Technologies on Virtualized RAN Midhaul Latency Budget: A Quantitative Experimental Evaluation," IEEE Communications Letters, vol. 23, no. 4, pp. 604-607, 2019.

[34] Y. Yoshida, "Mobile XHaul evolution: Enabling tools for a flexible 5G XHaul network," in Proc. Opt. Fiber Commun. Conf. Expo., San Diego, CA, USA, pp. 1-85, 2018.

[35] M. Habibi, M. Nasimi, B. Han, and H. Schotten, "A comprehensive survey of RAN architectures toward 5G mobile communication system," IEEE Access, vol. 7, pp. 70371-70421, 2019.

[36] H. Eran, D. Levi, L. Liss and M. Silverstein, "NFV acceleration: the role of the NIC," $8^{\text {th }}$ Workshop on System for Multi-core and Heterogeneous Architectures, 2018.

[37] S. Sarmiento, et al., "Optical Power Budget Enhancement in $50 \mathrm{~Gb} / \mathrm{s} \mathrm{IM-}$ DD PONs with NOMA CAP Modulation and SOA-based Amplification," 21th International Conference of Transparent Optical Network (ICTON), Angers, France, pp. 1-4, 2019.

[38] S. Sarmiento, et al., "Experimental Investigation of 50-90 Gb/s IM-DD NOMA-CAP Modulation for Short Range Optical Transmission Applications," 24th OptoElectronics and Communications Conference/International Conference on Photonics in Switching and Computing, Fukuoka, Japan, pp. 1-3, 2019.

[39] S. Sarmiento et al., "Optical Power Budget Enhancement in $50-90 \mathrm{~Gb} / \mathrm{s}$ IM-DD PONs With NOMA-CAP and SOA-Based Amplification," IEEE Photonics Technology Letters, vol. 32, no. 10, pp. 608-611, 15 May15, 2020.

[40] S. Sarmiento, et al., "Converged RoF-based mobile fronthaul and passive optical network with NOMA-CAP modulation format," SPIE Photonics West, San Francisco, USA, 2020.

[41] X. Tang, J. Zhou, M. Guo, J. Qi, F. Hu, Y. Qiao, Y. Lu, "40-Gb/s PAM4 with low-complexity equalizers for next-generation PON systems," Optical Fiber Technology, vol. 40, 2018, pp. 108-113.

[42] J. Gao, "Demonstration of the first 29dB Power Budget of 25Gb/s 4-PAM System without Optical Amlifier for Next Generation Access Network," in Proc. Optical Fiber Communications Conference (OFC), paper Th1I.2, Anaheim, 2016.

[43] S. D. Le, A. Lebreton, F. Saliou, Q. Deniel, B. Charbonier and P. Chanclou, "Up to $60 \mathrm{~km}$ bidirectional transmission of a 16 channels $\times 10$ Gb/s FDM-WDM PON based on self-seeded reflective semiconductor optical amplifiers," OFC 2014, San Francisco, CA, 2014, pp. 1-3.

[44] David Smalley, "Equalization Concepts: A Tutorial," Application Report, Texas Instruments, (1994).

[45] D. Visani, G. Tartarini, L. Tarlazzi and P. Faccin, "Transmission of UMTS and WIMAX Signals Over Cost-Effective Radio Over Fiber Systems," IEEE Microwave and Wireless Components Letters, vol. 19, no. 12, pp. 831-833, 2009.

[46] T. Kageyama, O. Muta and H. Gacanin, "Performance Analysis of OFDM With Peak Cancellation Under EVM and ACLR Restrictions," IEEE
Transactions on Vehicular Technology, vol. 69, no. 6, pp. 6230-6241, 2020.

[47] D. Wake and R. E. Schuh, "Measurement and simulation of W-CDMA signal transmission over optical fibre," IEEE Electronics Letters, vol. 36, no. 10, pp. 901-902, 11 May 2000

[48] S. Soerensen, "Optical beat noise suppression and power equalization in subcarrier multiple access passive optical networks by downstream feedback," IEEE Journal of Lightwave Technology, vol. 18, no. 10, pp. $1337-1347,2000$

[49] C. Desem, "Optical interference in subcarrier multiplexed systems with multiple optical carriers," IEEE J. Sel. Areas Comm. 8(7), 1290-1295 (1990).

[50] C. H. Chang, "Interference of multiple optical carriers in subcarriermultiplexed systems," IEEE Photon. Technol. Lett. 5(7), 848-850 (1993).

[51] ITU-T Recommendation, G.975.1 (2004).

[52] J. A. Altabas et al., "Real-Time 10 Gbps Polarization Independent Quasicoherent Receiver for NG-PON2 Access Networks," IEEE Journal of Lightwave Technology, vol. 37, no. 2, pp. 651-656, 2019. 